\title{
Usefulness of Early Videofluoroscopic Swallowing Study in Acute Stroke Patients With Dysphagia
}

\author{
Sang Beom Kim, MD, Sook Joung Lee, MD, Kyeong Woo Lee, MD, \\ Jong Hwa Lee, MD, Dong Won Kim, MD
}

Department of Physical Medicine and Rehabilitation, Dong-A University College of Medicine \& Busan-Ulsan Regional Cardiocerebrovascular Center, Busan, Korea

Objective To demonstrate the usefulness of early videofluoroscopic swallowing study (VFSS) and to investigate change patterns in dietary methods in stroke patients with dysphagia.

Methods The VFSS was performed within 7 days of stroke onset in neurologically stable patients. The patients were divided into three groups according to type of brain lesion: cortical lesion (CL), subcortical lesion (SCL), and brainstem/cerebellar lesion (BCL). Based on the VFSS results, this study investigated change patterns in feeding method and discrepancies in the aspiration risk predicted by the Water Swallowing Test (WST) and the VFSS. Complications, such as aspiration pneumonia, were also evaluated.

Results A total of 163 patients met the inclusion criteria and the VFSS was performed within 7 days of stroke. Patients considered at risk for aspiration (Penetration-Aspiration Scale [PAS] scores of 6 to 8) were found in all three groups using the VFSS (47.5\% of the CL group, 59.3\% of the SCL group, and $47.9 \%$ of the BCL group). After early VFSS, $79.7 \%$ of the patients were assessed to require restricted feeding methods. A $19.0 \%$ discrepancy was found between the WST and VFSS results. At 3-week follow-up after the VFSS, aspiration pneumonia was observed in 12 patients ( $7.4 \%$ ) with restricted feeding methods.

Conclusion Early VFSS during the acute period can facilitate determination of the most appropriate feeding method, and support effective dysphagia management for stroke patients.

Keywords Acute stroke, Dysphagia, Early videofluoroscopic swallowing study, Diet modification

Department of Physical Medicine and Rehabilitation, Dong-A University Hospital, Dong-A University College of Medicine, 26 Daesingongwon-ro, Seogu, Busan 49201, Korea. Tel: +82-51-240-5691, Fax: +82-51-254-8511, E-mail: flaehdrbs@naver.com

ORCID: Sang Beom Kim (http://orcid.org/0000-0002-5622-5933); Sook Joung Lee (http://orcid.org/0000-0002-6894-445X); Kyeong Woo Lee (http:// orcid.org/0000-0001-8785-5535); Jong Hwa Lee (http://orcid.org/0000-0003-2489-358X); Dong Won Kim (http://orcid.org/0000-0002-1856-2592).

() This is an open-access article distributed under the terms of the Creative Commons Attribution Non-Commercial License (http://creativecommons.org/ licenses/by-nc/4.0) which permits unrestricted noncommercial use, distribution, and reproduction in any medium, provided the original work is properly cited. Copyright $\odot 2018$ by Korean Academy of Rehabilitation Medicine 


\section{INTRODUCTION}

Stroke is a disease associated with various cerebrovascular symptoms, such as motor weakness, paresthesia, balance problems, gait disturbance, cognitive impairment, language problems, and swallowing disorders [1]. Swallowing problems have been reported in $37 \%-78 \%$ of stroke patients depending on the timing of the screening and the assessments used [2,3]. Post-stroke dysphagia is independently associated with pneumonia, which develops in more than one-third of aspirators, and 3.8\% of stroke patients with pneumonia die due to this complication [4]. Importantly, early detection of swallowing deficiencies is associated with lower pneumonia rates [5].

Formal dysphagia screening protocols (DSP) can significantly lower pneumonia risk and improve general outcomes $[6,7]$. Thus, most stroke patients' feeding methods are determined by formal DSP within 24 hours of stroke onset [8]. However, in some cases, the acute stoke patient with dysphagia has a nasogastric (NG) tube left in place for an extended period, even if the patient can eat orally, and the NG tube could be removed. Such cases occur when the patient's level of consciousness has changed so that the patient is alert, when aphasia or cognitive impairment has improved, when the swallowing problem has resolved due to natural stroke recovery, or when an NG tube was originally inserted due to false-positive screening results, as in episodic aspiration. Long-term NG tube insertion may cause various complications such as nasal wing lesions, chronic sinusitis, gastro-esophageal reflux, and aspiration pneumonia [9]. Removing the NG tube and switching to a more relaxed feeding method is beneficial for such patients.

In contrast, dysphagia screening tests may yield falsenegative results. These screening tests have been limited to the detection of residual volume and silent aspiration, so acute stroke patients with dysphagia have sometimes been given normal regular diets (NRD) even if they have silent aspiration symptoms. In such cases, a more restrictive feeding method should be adopted.

Diverse methods are used to detect dysphagia in stroke patients. Salivagram, videofluoroscopic swallowing study (VFSS), and fiberoptic endoscopic evaluation of swallowing (FEES) are among the most frequently used methods. The VFSS has limitations; it cannot be implemented when the patient cannot maintain a sitting position or has a poor state of consciousness. It also has advantages; it allows bolus flow, muscle movement, and the relationship of anatomy with aspiration to be observed in real time, and the test outputs do not differ much according to the skill of the person administering the test compared to FEES [10-12]. For these reasons, the VFSS is widely used at points of care and in many studies.

Dysphagia screening tests and the VFSS have exhibited discrepancies in sensitivity and specificity at predicting the risk of aspiration. Sensitivity and specificity have been reported to be $39 \%$ and $82 \%$, respectively, in screening test and at $57.6 \%$ and $82.5 \%$, respectively, in VFSS [13].

In the acute stroke phase, sudden changes in neurologic status such as rapid recovery or deterioration can occur. Thus, re-evaluation is necessary to modify feeding methods appropriately and to manage dysphagia in acute stroke patients. However, no definite guidelines for the dysphagia re-evaluation period in acute stroke patients exist. We hypothesizes that intensive re-evaluation within 7 days of stroke onset is needed due to the high incidence of aspiration and rapid change of neurologic status during the acute period of stroke. Early performance of the VFSS after stroke, therefore, could facilitate the determination of appropriate feeding methods and improve function and quality of life for stroke patients.

This study investigated the usefulness of early VFSS and the change patterns in feeding methods in acute stroke patients with dysphagia.

\section{MATERIALS AND METHODS}

The present study was conducted with patients who visited the researchers' stroke center between January 2015 and December 2016.

Each patient's feeding method was determined using DSP at the hospital's stroke center within 24 hours of arrival (Fig. 1). The stroke center implemented the DSP described Massey and Jedlicka [14] through the following process. For the patient with a mental status of less than mildly drowsy, non-oral feeding methods (NOFM) such as NG tubes were implemented. When the patient was unable to cooperate despite alert mental status (e.g., a patient in whom the oral phase had not started or been maintained), the current feeding method was discontinued, and an NG tube was inserted.

Bedside swallowing screening tests were conducted be- 


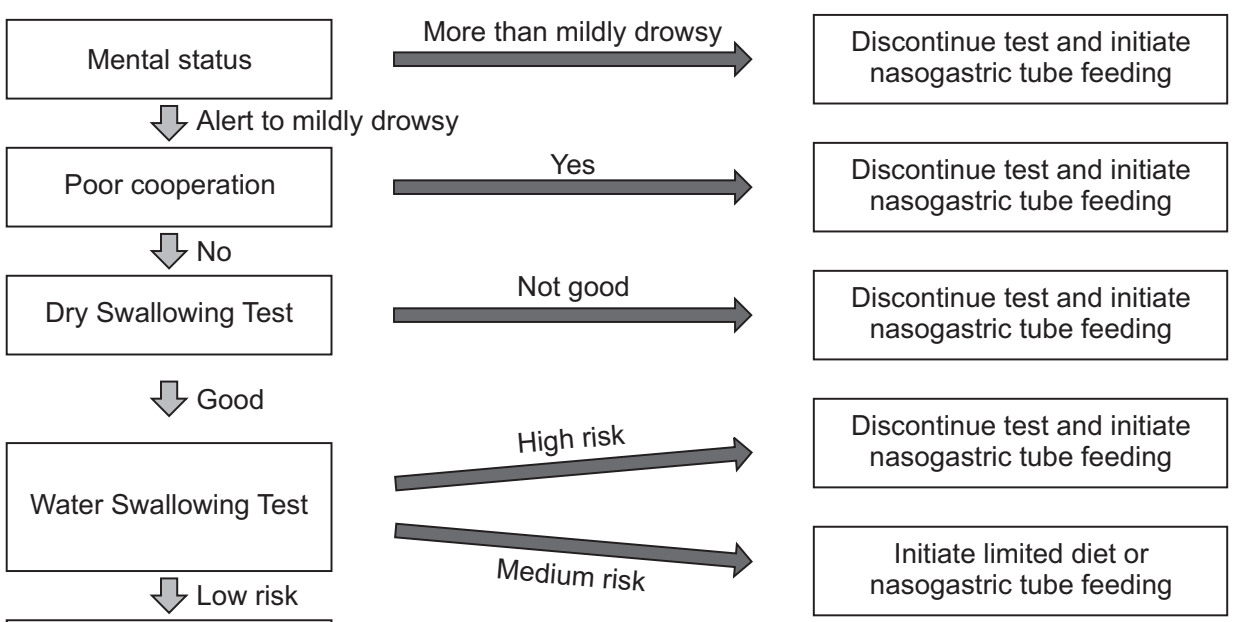

Initiate limited diet

or normal regular diet
Fig. 1. Dysphagia screening protocol. Patients' feeding methods were determined using the hospital's dysphagia screening protocol within 24 hours of arrival. fore the VFSS. First, in a dry swallowing test, the patient was asked to swallow their own saliva, and their reactions were observed. For the patients who showed abnormalities during this test, an NG tube was inserted. For the patients who showed no abnormal findings in the dry swallowing test, a Water Swallowing Test (WST) was conducted. The patient was instructed to swallow $50 \mathrm{~mL}$ of water and was observed for involuntary coughing, drooling, voice changes and swallowing delays of 2 seconds or more. The number of observed items was converted into a risk level. Cases with 2 or more observed abnormal items were defined as high risk, cases with 1 observed abnormal item were defined as medium risk, and cases in which no abnormal items were observed were defined as low risk. For the patients in the high-risk category, their current feeding methods were discontinued, and an NG tubes were inserted. In the case of the medium-risk patients, the current feeding methods were modified to a limited diet (LD) or NG tubes. In the case of the low-risk patients, the current feeding methods were modified to a LD, or the NRD was maintained, as determined by the clinician.

After primary stroke treatment, the VFSS was performed in neurologically stable patients along with rehabilitation therapy within 7 days of the onset of stroke in the following cases: NG-tube-insertion patients with recovering mentality and improving cooperation, patients who complained of aspiration symptoms or residual sensations after swallowing, patients who showed dysarthria, and patients who had brainstem lesions.
Cases excluded from the study where those in which the patients had not recovered mentality, the patients were not cooperative to the extent that the oral phase had not started (Mini-Mental Status Examination [MMSE] $<10$ ), the patients had bilateral hemispheric lesions, the patients had dysphagia symptoms before stroke onset, aspiration pneumonia occurred before performance of the initial VFSS, or the patients had recurrent stroke.

The patients were divided into three groups according to type of brain lesion: cortical lesion (CL), subcortical lesion (SCL), and brainstem/cerebellar lesion (BCL). That the severity and the prognosis of dysphagia vary with types of brain lesions has been demonstrated in previous studies [15-17].

Before the VFSS was performed, the Korean version of the MMSE (K-MMSE) was conducted to evaluate the patients' cognitive status. The Korean version of the Modified Barthel Index (K-MBI) was used to measure the patient's general functions. The Dysphagia Outcome and Severity Scale (DOSS) was used to evaluate the degree of dysphagia.

The VFSS was performed using the revised Logemann protocol, in which the patient was in a sitting position so that the lateral view could be observed during the swallowing process. With barium as a contrast medium, the patient was asked to swallow a thick liquid, pureed food, a semisolid food, a solid food, and a thin liquid three times each in order. Any observed abnormalities were noted $[11,18]$.

Using the VFSS, the oral and pharyngeal phases, the 
total Functional Dysphagia Scale (FDS), and the Penetration-Aspiration Scale (PAS) were obtained. The FDS was developed to quantify the severity of dysphagia and correlates well with the American Speech-Language-Hearing Association-National Outcome Measurement System criteria. The FDS consists of 11 items with weighted values representing 4 oral functions and 7 pharyngeal functions that can be observed through the VFSS $[18,19]$. The PAS evaluates airway invasion and has a maximum score of 8 points with 6-8 points indicating aspiration [20].

In addition, peak cough flows (PCF) were obtained using a digital peak-flow meter (MicroPeak; Micro Medical Ltd., Rochester, UK). The PCF measures voluntary coughing ability. The patient was instructed to cough as intensely as possible three times consecutively, and the highest value shown in the digital peak-flow meter was selected as the result [18].

The VFSS was monitored to detect the required changes in feeding methods and any discrepancies with the screening test. The VFSS images were analyzed by two rehabilitation specialists using FDS and PAS. NRD was recommended when there were no abnormalities in solid and liquid diet. For the patients with severely delayed oral transit time and those who had shown large amount of silent aspiration even though small amounts of thick liquid or pureed food were tried, NOF was recommended. Except for the aforementioned cases, LD was recom- mended according to the risk of aspiration, the amount of residue and oral transit time.

Incidences of aspiration pneumonia were evaluated for up to 3 weeks after stroke onset. Abnormal patients received individualized dysphagia therapy based on the VFSS results.

Windows SPSS version 18.0 (SPSS Inc., Chicago, IL, USA) was employed for the statistical analyses. To detect statistically significant differences among the three groups, chi-square tests and non-parametric KruskalWallis tests were performed for categorical-type data (gender, type of stroke, laterality of lesion, screening test, current feeding method, laterality of lesion, and PAS $\geq 6$ ) and for continuous-type data (age, MMSE, K-MBI, DOSS, FDS \& PAS, PCF, and test days from stroke onset), respectively. The statistical significance level was set at $\mathrm{p}<0.05$.

The study protocol was approved by the Institutional Review Board of Dong-A University Hospital (No. DAUHIRB-17-101) and all the participants provided written informed consent.

\section{RESULTS}

This study enrolled 163 patients who met the inclusion criteria, and the VFSS was performed within 7 days of stroke. The CL group had 61 patients, the SCL group 54 patients, and the BCL group 48 patients (Fig. 2).

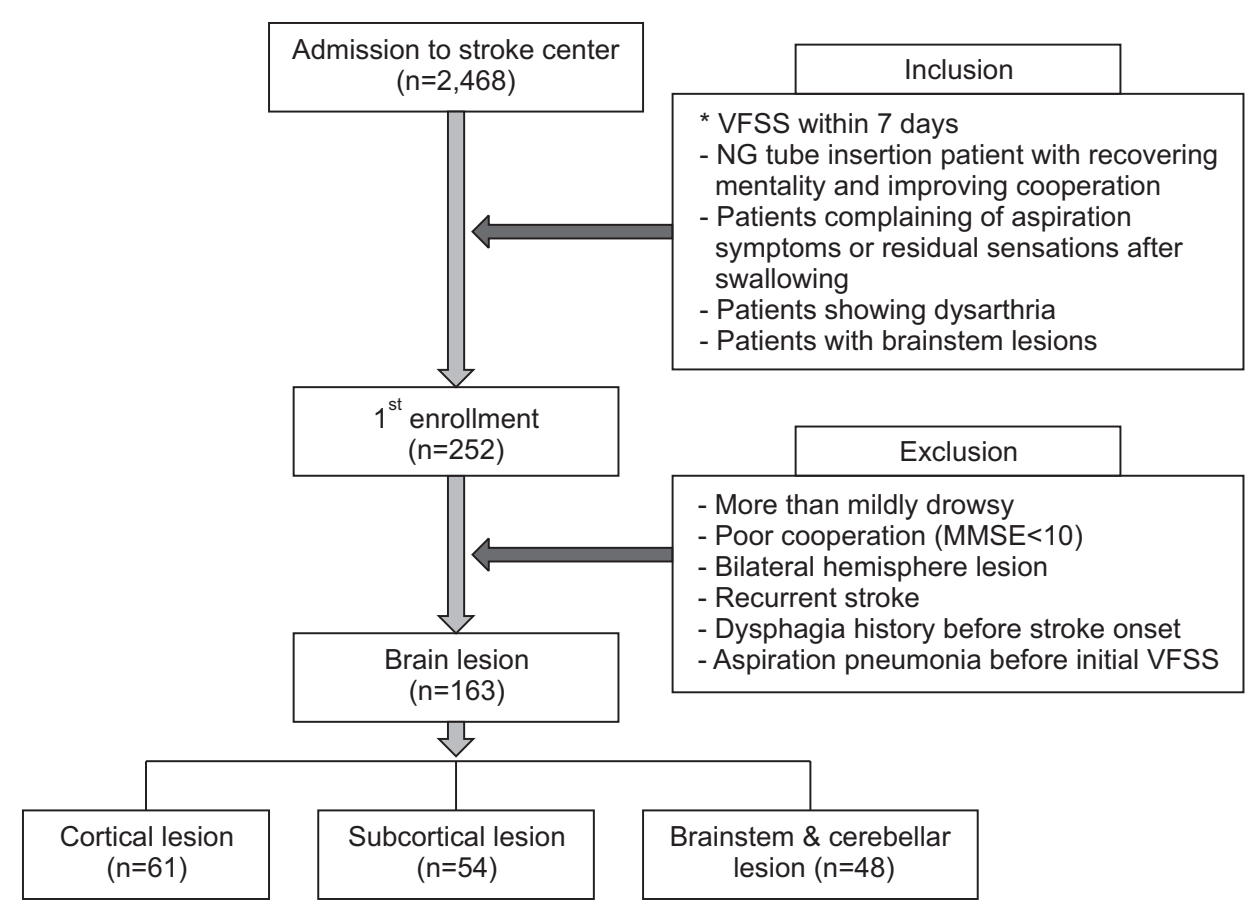

Fig. 2. Flowchart of the study design. VFSS, videofluoroscopic swallowing study; NG, nasogastric; MMSE, Mini-Mental Status Examination. 
Table 1. Demographic characteristics of patients included in this study

\begin{tabular}{|c|c|c|c|c|}
\hline & CL $(n=61)$ & SCL $(n=54)$ & BCL $(n=48)$ & p-value \\
\hline Age (yr) & $68.48 \pm 11.03$ & $65.72 \pm 13.50$ & $66.46 \pm 12.05$ & 0.571 \\
\hline Gender & & & & 0.189 \\
\hline Male & 39 & 28 & 33 & \\
\hline Female & 22 & 26 & 15 & \\
\hline Type of stroke & & & & 0.000 \\
\hline Ischemic & 59 & 32 & 45 & \\
\hline Hemorrhagic & 2 & 22 & 3 & \\
\hline Laterality of lesion & & & & 0.031 \\
\hline Left hemisphere & 38 & 21 & 28 & \\
\hline Right hemisphere & 23 & 33 & 20 & \\
\hline K-MMSE & $20.87 \pm 6.10$ & $21.54 \pm 5.95$ & $23.10 \pm 5.60$ & 0.183 \\
\hline K-MBI & $55.07 \pm 25.84$ & $37.59 \pm 24.34$ & $46.79 \pm 27.24$ & $0.003^{*}$ \\
\hline \multicolumn{5}{|l|}{ Feeding method } \\
\hline Non-oral feeding & $18(29.5)$ & $10(18.5)$ & $13(27.1)$ & 0.335 \\
\hline Limited diet & $17(27.9)$ & $24(44.4)$ & $14(29.2)$ & - \\
\hline NRD & $26(42.6)$ & $20(37.0)$ & $21(43.8)$ & - \\
\hline \multicolumn{5}{|c|}{ Bedside water screening test } \\
\hline Low risk & $19(31.1)$ & $24(44.4)$ & $22(45.8)$ & 0.210 \\
\hline Medium/high risk & $42(68.9)$ & $30(55.6)$ & $26(54.2)$ & - \\
\hline DOsS & $4.03 \pm 2.07$ & $3.93 \pm 1.77$ & $3.21 \pm 2.12$ & 0.862 \\
\hline
\end{tabular}

Values are presented as mean \pm standard deviation or number (\%).

CL, cortical lesion group; SCL, subcortical lesion group; BCL, brainstem/cerebellar lesion group; K-MMSE, Korean version of the Mini-Mental Status Examination; K-MBI, Korean version of the Modified Barthel Index; NRD, normal regular diet; DOSS, Dysphagia Outcome and Severity Scale.

${ }^{*} \mathrm{p}<0.05$ by the Kruskal-Wallis and the chi-square test.

Table 1 shows the patients' baseline demographic characteristics. The patients in the CL group ( $\mathrm{n}=61)$ had an average age of $68.48 \pm 11.03$ years, the patients in the SCL group ( $\mathrm{n}=54) 65.72 \pm 13.50$ years, and the patients in the BCL group $(\mathrm{n}=48) 66.46 \pm 12.05$ years. Of the 163 patients, 100 were male and 63 were female. The sample included 136 patients who had suffered hemorrhagic stroke and 27 patients who had suffered ischemic stroke; 87 patients had been affected by left hemisphere lesions and 76 by right hemisphere lesions. The K-MBI scores of the CL, SCL, and BCL groups were $55.07 \pm 25.84,37.59 \pm 24.34$, and $46.79 \pm 27.24$, respectively. The CL group had the highest K-MBI scores, and the differences in the scores were significant (Table 1).

The VFSS was conducted with the patients in the CL, SCL, and BCL groups 4.52 $\pm 1.45,5.20 \pm 1.41$, and $4.71 \pm 1.44$ days, respectively, after stroke onset. Differences were observed at the time of the VFSS performance after stroke onset among the three groups. However, no statistically significant differences in FDS or PAS were observed among the three groups. In the VFSS performed within 7 days of stroke onset, PAS scores of at least 6 points were recorded for 29 patients in the CL group (47.5\%), 32 patients in the SCL group (59.3\%), and 23 patients in the BCL group (47.9\%). These differences among the three groups were not statistically significant (Table 2).

The post-VFSS changes in feeding methods according to stroke lesion type are shown in Table 3. Of the patients enrolled in the study, $33(20.3 \%)$ were able to maintain a NRD within 7 days after the VFSS (Table 3 ).

Compared to the feeding methods used before performance of the VFSS, feeding restrictions could be relaxed in 44 patients $(27.0 \%)$, while feeding had to be further restricted in 61 patients (37.4\%). Feeding restrictions such as changing from an NRD before stroke to an LD were observed in 15 patients (24.6\%) in the CL group, 14 patients 
Table 2. Results of swallowing tests according to type of stroke lesion

\begin{tabular}{lcccc}
\hline & CL $(\mathbf{n = 6 1})$ & SCL $(\mathbf{n}=\mathbf{5 4})$ & BCL $(\mathbf{n}=\mathbf{4 8})$ & p-value \\
\hline Test days from stroke onset & $4.76 \pm 1.34$ & $5.15 \pm 1.27$ & $4.50 \pm 1.73$ & $0.031^{*}$ \\
VFSS score & & & & 0.782 \\
FDS-total & $24.82 \pm 13.18$ & $25.72 \pm 12.00$ & $26.77 \pm 12.16$ & 0.702 \\
FDS-oral & $0.77 \pm 2.43$ & $1.20 \pm 3.37$ & $0.65 \pm 2.34$ & 0.761 \\
FDS-pharyngeal & $25.03 \pm 14.81$ & $24.52 \pm 10.68$ & $26.13 \pm 11.32$ & 0.349 \\
PAS & $4.92 \pm 2.81$ & $5.63 \pm 2.54$ & $5.27 \pm 2.50$ & 0.381 \\
PAS $\geq 6$ & $29(47.5)$ & $32(59.3)$ & $23(47.9)$ & 0.309 \\
PCF & $204.00 \pm 138.79$ & $177.74 \pm 134.50$ & $208.56 \pm 110.50$ & \\
\hline
\end{tabular}

Values are presented as mean \pm standard deviation or number (\%).

CL, cortical lesion group; SCL, subcortical lesion group; BCL, brainstem/cerebellar lesion group; VFSS, videofluoroscopic swallowing study; FDS, Functional Dysphagia Scale; PAS, Penetration-Aspiration Scale; PCF, peak cough flow. ${ }^{*} \mathrm{p}<0.05$ by the Kruskal-Wallis and the chi-square test.

Table 3. Changes in feeding methods after early VFSS

\begin{tabular}{|c|c|c|c|c|c|c|c|}
\hline & \multicolumn{2}{|c|}{$C L(n=61)$} & \multicolumn{2}{|c|}{$\operatorname{SCL}(n=54)$} & \multicolumn{2}{|c|}{ BCL $(n=48)$} & Total $(n=163)$ \\
\hline & Before & After & Before & After & Before & After & After \\
\hline NOF & $18(29.5)$ & $6(9.8)$ & $10(18.5)$ & $10(18.5)$ & $13(27.1)$ & $8(16.7)$ & $24(14.7)$ \\
\hline LD & $17(27.9)$ & $41(67.2)$ & $24(44.5)$ & $34(63.0)$ & $14(29.2)$ & $31(64.6)$ & $106(65.0)$ \\
\hline NRD & $26(42.6)$ & $14(22.9)$ & $20(37.0)$ & $10(18.5)$ & $21(43.7)$ & $9(18.8)$ & $33(20.3)$ \\
\hline
\end{tabular}

Values are presented as number (\%).

VFSS, videofluoroscopic swallowing study; CL, cortical lesion group; SCL, subcortical lesion group; BCL, brainstem/ cerebellar lesion group; NOF, non-oral feeding; LD, limited diet; NRD, normal regular diet.

Table 4. Change patterns in feeding methods after early VFSS

\begin{tabular}{lcccc}
\hline & CL (n=61) & SCL (n=54) & BCL (n=48) & Total (n=163) \\
\hline Relaxing control of the feeding method & & & & \\
NOF $\rightarrow$ LD & $13(21.3)$ & $8(14.8)$ & $12(25.0)$ & \\
LD $\rightarrow$ NRD & $3(4.9)$ & $4(7.4)$ & $4(8.3)$ & \\
Total & $16(26.2)$ & $12(22.2)$ & $16(33.3)$ & $44(27.0)$ \\
\hline Restricting control of the feeding method & & & & \\
NRD $\rightarrow$ LD & $15(24.6)$ & $14(25.9)$ & $16(33.3)$ & \\
LD $\rightarrow$ NOF & $1(1.6)$ & $8(14.8)$ & $7(14.6)$ & \\
Total & $16(26.2)$ & $22(40.7)$ & $23(47.9)$ & $61(37.4)$ \\
\hline No change in feeding method & $29(47.6)$ & $20(37.1)$ & $9(18.8)$ & $58(35.6)$ \\
\hline
\end{tabular}

Values are presented as number (\%).

VFSS, videofluoroscopic swallowing study; CL, cortical lesion group; SCL, subcortical lesion group; BCL, brainstem/ cerebellar lesion group; NOF, non-oral feeding; LD, limited diet; NRD, normal regular diet.

(25.9\%) in the SCL group, and 16 patients $(33.3 \%)$ in the BCL group. There was no change in feeding method after the initial DSP and VFSS performance in 58 patients (35.6\%) (Table 4).

Discrepancies in aspiration risk predicted by WST and the VFSS were confirmed in the following two cases. First, PAS score higher than 6 were observed on the VFSS when the WST produced no abnormal findings. Second, the VFSS showed no abnormal findings, while the WST indicated more than one abnormal finding. Discrepan- 
Table 5. Discrepancies in aspiration risk between WST and VFSS results

\begin{tabular}{|c|c|c|c|c|}
\hline & $\underset{(n=61)}{C L}$ & $\underset{(n=54)}{\text { SCL }}$ & $\begin{array}{c}\text { BCL } \\
(n=48)\end{array}$ & $\begin{array}{c}\text { Total } \\
(n=163)\end{array}$ \\
\hline WST (non-specific findings) and VFSS (PAS $\geq 6$ ) & 9 & 5 & 7 & 21 \\
\hline WST (abnormal sign $\geq 1$ ) and VFSS (non-specific findings) & 4 & 4 & 2 & 10 \\
\hline Discrepancies in aspiration risk between WST and VFSS & $13(21.3)$ & $9(16.7)$ & $9(18.6)$ & $31(19.0)$ \\
\hline
\end{tabular}

Values are presented as number (\%).

WST, Water Swallowing Test; VFSS, videofluoroscopic swallowing study; CL, cortical lesion group; SCL, subcortical lesion group; BCL, brainstem/cerebellar lesion group.

cies were observed in 13 of 61 (21.3\%), patients in the CL group, 9 of 54 (16.7\%), patients in the SCL group, and 9 of $48(18.6 \%)$ patients in the BCL group. Thus, in the total sample of 163 cases, 31 (19.0\%) showed discrepancies (Table 5).

During the 3-week follow-up period after the VFSS, aspiration pneumonia was observed in 3 patients (5.0\%) in the CL group, 5 patients (9.3\%) in the SCL group, and 4 patients $(8.3 \%)$ in the BCL group, for a total of 12 of the 163 patients (7.4\%). Aspiration pneumonia was observed in some patients who required restricted dietary methods. Among the patients who had aspiration pneumonia, the changes in dietary restrictions in each group can be summarized as follows: in the CL group, 1 patient changed from LD to NOFM and 2 patients from NRD to NOFM. Five patients in the SCL group changed from NRD to LD. In the BCL group, 3 patients changed from NRD to LD and 1 patient changed from LD to NOFM.

\section{DISCUSSION}

The purpose of the present study was to assess the usefulness of performing early VFSS within 7 days of stroke onset and to observe the change patterns in feeding methods. Compared to that before stroke onset, NRDs could be maintained in $22.9 \%$ of the patients in the CL group, $18.5 \%$ of the patients in the SCL group, and $18.8 \%$ of the patients in the BCL group. Of the 163 patients in the study, 33 (20.3\%) maintained NRDs. In other words, approximately $80 \%$ of the patients had to alter their method of nutritional intake after acute stroke. This level is similar to levels of $37 \%-78 \%$, reported in previous studies, depending on the assessment timing and the methods of dysphagia measurement after stroke [2,3].

After early VFSS, feeding methods had to be modified for $64.4 \%$ of patients, as determined by DSP. In these cases, the feeding method determined with DSP could be relaxed in $27 \%$ of cases. However, $37.4 \%$ required more restrictions than the feeding method determined with DSP. Comparing changes from DSP in individual groups, feeding methods were maintained without any change in $47.6 \%$ of the patients in the CL group, $37.1 \%$ of the patients in the SCL group, and only $18.8 \%$ of the patients in the BCL group, which saw the greatest change. These findings indicate that DSP has limitations for measuring silent aspiration and residual volumes and that combining VFSS, which can evaluate such factors, with DSP at an early stage, can more effectively manage stroke patients' dysphagia.

A large-sample meta-analysis was conducted to determine the association between neuroanatomical lesions and stroke dysphagia using MRI. It found that available evidence was limited to infratentorial strokes. Among these cases, pontine and medullary lesions were more frequent than cerebellar or midbrain lesions [21]. Early VFSS, therefore, should be performed in cases of BCL lesions, especially if the lesions are pontine or medullary. In such cases, the frequency of dysphagia is high and changes in feeding methods could be significant, even if there are no significant abnormal findings after DSP as observed in the present study.

The WST is a screening tool commonly used in DSP. Differences between the WST and the VFSS results were observed in 31 (19.0\%) of the 163 patients. This finding indicates that the WST has limited ability to detect silent aspiration, although it is highly sensitive.

The timing of implementation after stroke can be considered as another factor that affects the discrepancies in aspiration risk between the WST and the VFSS results. The WST is usually implemented within 24 hours after stroke onset. In the present study, the timing of the VFSS differed in each group, with $4.76 \pm 1.34$ days in the CL 
group, $5.15 \pm 1.27$ days in the SCL group, and $4.50 \pm 1.73$ days in the BCL group. Prevalence of dysphagia has been reported to be approximately $42 \%-67 \%$ within 3 days after stroke onset $[22,23]$. Although recovery from mildmoderate dysphagia was made within 7 days in most cases, aspiration was continuously observed in approximately $50 \%$ of the patients with dysphagia [24]. Timing, therefore, does not have large impact on the outcome as long as the VFSS is implemented within 7 days.

In the present study, after early VFSS, PAS scores of 6-8 points were observed in $84(51.5 \%)$ patients and the onset of aspiration pneumonia was observed in $12(7.4 \%)$ of the 163 patients. Aspiration pneumonia independently increases the mortality risk. It is one of the main causes of death in the first few days and weeks after stroke [25]. In previous studies, the frequency of aspiration pneumonia has been reported to be $6 \%-10 \%$ [26]. In the present study, the onset of aspiration pneumonia was observed in $7.4 \%$ of the patients lower than $10 \%$. This result can be attributed to the patient selection criteria of the present study that excluded bed ridden patients who could not maintain a sitting position, patients who were unable to cooperate, patients with MMSE scores of less than 10 points, and patients who were not mentally alert.

During the 3-week follow-up period, aspiration pneumonia was observed only in the patients for whom feeding methods had to be restricted. Strong evidence has been found for an association between the risk of stroke-related pneumonia and delays in carrying out comprehensive dysphagia assessment [27]. Accordingly, if feeding methods are maintained without early VFSS, the incidence of aspiration pneumonia should have been higher in the $37.4 \%$ of patients who required feeding restrictions. As shown, early VFSS can be a useful tool in the management of stroke patients, and a combination of proper DSP and early VFSS is essential for management of dysphagia. However, there are no established guidelines for the optimal time to perform VFSS after stroke.

The present study, therefore, complemented DSPs with the use of objective tests such as VFSS to enable appropriate modification of feeding methods for patients placed under unnecessary restrictions despite improvement in their condition and the early initiation of appropriate swallowing therapy. This combination of methods can help acute stage stroke patients achieve improved functional outcomes and quality of life. The present study examined the patterns of changes in the feeding methods of early stroke patients and, based on these results, supported the usefulness of early VFSS. However, the present study does have several limitations.

First, the VFSS cannot be performed for patients who cannot maintain a sitting position or cannot cooperate. Therefore, such patients were excluded from this study. Second, although the patients were divided into different groups according to brain lesion type in the study design, not all brain lesions were observed because bihemispheric stroke was not observed. Third, the study design included brainstem lesions and cerebellar lesions in the same group. A meta-analysis by Flower et al. [21] has found evidence that dysphagia only exists in infratentorial lesions, including those in the cerebellum. A number of previous studies have classified patients into two groups as supratentorial lesions (cortex, subcortex) and infratentorial lesions. Hence, the present study included brainstem lesions and cerebellar lesions in the same group. However, close scrutiny of meta-analysis results indicates that there is only a weak relation between cerebellar lesions and dysphagia in infratentorial lesions [21]. Therefore, dysphagia risk might have been underestimated because total number of cases in the BCL group was increased following the inclusion of cerebellar lesions. Fourth, selection bias might exist in this study. More high-risk patients were included in the CL group and the SCL group than those in the BCL group because this study included all patients with brainstem lesions. Finally, the three groups showed significant differences in the initial K-MBI scores. Such differences in severity might have affected the VFSS findings. In addition, the occurrence of aspiration pneumonia cannot be eliminated. Further studies that compensate for these limitations, therefore, are necessary.

In conclusion, this study aimed to demonstrate the usefulness of performing early VFSS within 7 days of stroke onset and found that more than two-thirds of the patients required changes in their feeding methods after evaluation with VFSS. This study included patients whose consciousness level or compliance was improved after using NG tubes for feeding after performance of DSPs within 24 hours. Moreover, the VFSS was performed within 7 days when the patient complained about aspiration symptoms or a stuffed feeling in the throat while eating food, when dysarthria presented, or when the patient 
had a brainstem lesion. Changes in swallowing function during the acute stroke phase can be especially dynamic in these patients. Implementation of early VFSS not only can facilitate a more appropriate dietary scheme, but also can contribute to effective dysphagia management. Early performance of the VFSS, therefore, can improve stroke patients' functionality and quality of life and can contribute to prevention of aspiration pneumonia.

\section{CONFLICT OF INTEREST}

No potential conflict of interest relevant to this article was reported.

\section{REFERENCES}

1. Axelsson K, Norberg A, Asplund K. Eating after a stroke: towards an integrated view. Int J Nurs Stud 1984;21:939.

2. Mann G, Hankey GJ, Cameron D. Swallowing function after stroke: prognosis and prognostic factors at 6 months. Stroke 1999;30:744-8.

3. Martino R, Foley N, Bhogal S, Diamant N, Speechley M, Teasell R. Dysphagia after stroke: incidence, diagnosis, and pulmonary complications. Stroke 2005; 36:2756-63.

4. Katzan IL, Cebul RD, Husak SH, Dawson NV, Baker DW. The effect of pneumonia on mortality among patients hospitalized for acute stroke. Neurology 2003;60:620-5.

5. Doggett DL, Tappe KA, Mitchell MD, Chapell R, Coates V, Turkelson CM. Prevention of pneumonia in elderly stroke patients by systematic diagnosis and treatment of dysphagia: an evidence-based comprehensive analysis of the literature. Dysphagia 2001; 16:279-95.

6. Hinchey JA, Shephard T, Furie K, Smith D, Wang D, Tonn S, et al. Formal dysphagia screening protocols prevent pneumonia. Stroke 2005;36:1972-6.

7. Middleton S, McElduff P, Ward J, Grimshaw JM, Dale S, D'Este C, et al. Implementation of evidence-based treatment protocols to manage fever, hyperglycaemia, and swallowing dysfunction in acute stroke (QASC): a cluster randomised controlled trial. Lancet 2011;378: 1699-706.

8. Alberts MJ, Hademenos G, Latchaw RE, Jagoda A,
Marler JR, Mayberg MR, et al. Recommendations for the establishment of primary stroke centers. Brain Attack Coalition. JAMA 2000;283:3102-9.

9. Gomes CA Jr, Lustosa SA, Matos D, Andriolo RB, Waisberg DR, Waisberg J. Percutaneous endoscopic gastrostomy versus nasogastric tube feeding for adults with swallowing disturbances. Cochrane Database Syst Rev 2010;11:CD008096.

10. Heckert KD, Komaroff E, Adler U, Barrett AM. Postacute reevaluation may prevent dysphagia-associated morbidity. Stroke 2009;40:1381-5.

11. Palmer JB, Kuhlemeier KV, Tippett DC, Lynch C. A protocol for the videofluorographic swallowing study. Dysphagia 1993;8:209-14.

12. Smithard DG. Dysphagia management and stroke units. Curr Phys Med Rehabil Rep 2016;4:287-94.

13. Smith Hammond CA, Goldstein LB, Horner RD, Ying J, Gray L, Gonzalez-Rothi L, et al. Predicting aspiration in patients with ischemic stroke: comparison of clinical signs and aerodynamic measures of voluntary cough. Chest 2009;135:769-77.

14. Massey R, Jedlicka D. The Massey Bedside Swallowing Screen. J Neurosci Nurs 2002;34:252-3.

15. Han DS, Chang YC, Lu CH, Wang TG. Comparison of disordered swallowing patterns in patients with recurrent cortical/subcortical stroke and first-time brainstem stroke. J Rehabil Med 2005;37:189-91.

16. Gonzalez-Fernandez M, Kleinman JT, Ky PK, Palmer JB, Hillis AE. Supratentorial regions of acute ischemia associated with clinically important swallowing disorders: a pilot study. Stroke 2008;39:3022-8.

17. Galovic M, Leisi N, Muller M, Weber J, Abela E, Kagi G, et al. Lesion location predicts transient and extended risk of aspiration after supratentorial ischemic stroke. Stroke 2013;44:2760-7.

18. Lee SJ, Lee KW, Kim SB, Lee JH, Park MK. Voluntary cough and swallowing function characteristics of acute stroke patients based on lesion type. Arch Phys Med Rehabil 2015;96:1866-72.

19. Han TR, Paik NJ, Park JW. Quantifying swallowing function after stroke: a functional dysphagia scale based on videofluoroscopic studies. Arch Phys Med Rehabil 2001;82:677-82.

20. Rosenbek JC, Robbins JA, Roecker EB, Coyle JL, Wood JL. A penetration-aspiration scale. Dysphagia 1996;11: 93-8. 
21. Flowers HL, Skoretz SA, Streiner DL, Silver FL, Martino R. MRI-based neuroanatomical predictors of dysphagia after acute ischemic stroke: a systematic review and meta-analysis. Cerebrovasc Dis 2011;32:110.

22. Perry L, Love CP. Screening for dysphagia and aspiration in acute stroke: a systematic review. Dysphagia 2001;16:7-18.

23. Kidd D, Lawson J, Nesbitt R, MacMahon J. The natural history and clinical consequences of aspiration in acute stroke. QJM 1995;88:409-13.

24. ECRI Health Technology Assessment Group. Diagnosis and treatment of swallowing disorders (dysphagia) in acute-care stroke patients. Evid Rep Technol Assess
(Summ) 1999;8:1-6.

25. Vernino S, Brown RD Jr, Sejvar JJ, Sicks JD, Petty GW, O'Fallon WM. Cause-specific mortality after first cerebral infarction: a population-based study. Stroke 2003;34:1828-32.

26. Westendorp WF, Nederkoorn PJ, Vermeij JD, Dijkgraaf MG, van de Beek D. Post-stroke infection: a systematic review and meta-analysis. BMC Neurol 2011;11:110.

27. Bray BD, Smith CJ, Cloud GC, Enderby P, James M, Paley $\mathrm{L}$, et al. The association between delays in screening for and assessing dysphagia after acute stroke, and the risk of stroke-associated pneumonia. J Neurol Neurosurg Psychiatry 2017;88:25-30. 